\title{
Wiman's formula for a second order dynamic equation
}

\author{
Lynn Erbe ${ }^{1}$, Gro Hovhannisyan ${ }^{2 *}$ and Alan Peterson ${ }^{1}$
}

${ }^{\text {"Correspondence: }}$

ghovhann@kent.edu

${ }^{2}$ Department of Mathematics, Kent

State University at Stark, 6000 Frank

Ave. NW, Canton, OH 44720-7599,

USA

Full list of author information is

available at the end of the article

\section{Abstract}

We derive Wiman's asymptotic formula for the number of generalized zeros of (nontrivial) solutions of a second order dynamic equation on a time scale. The proof is based on the asymptotic representation of solutions via exponential functions on a time scale. By using the Jeffreys et al. approximation we prove Wiman's formula for a dynamic equation on a time scale. Further we show that using the Hartman-Wintner approximation one can derive another version of Wiman's formula. We also prove some new oscillation theorems and discuss the results by means of several examples. MSC: 34E20; 34N05

Keywords: dynamic equation on a time scale; oscillation theory; number of zeros; asymptotic representation of solutions; Jeffreys, Wentzel, Kramers and Brillouin approximation

\section{Introduction}

Consider the equation

$$
u^{\Delta \Delta}(t)+\frac{u(t)}{\left(w^{\sigma}(t) w(t)\right)^{2}}=0, \quad t \geq t_{0}
$$

on $\left[t_{0}, t\right)_{\mathbb{T}}=\mathbb{T} \cap\left[t_{0}, t\right)$, where $\mathbb{T}$ is a time scale (a closed nonempty subset of the real numbers $\mathbb{R})$. Let $N\left(t_{0}, t\right)$ be the number of generalized zeros of solutions of (1.1) on $\mathbb{T}_{0}$. The classical result of Wiman [1] for the continuous time scale case states that if (1.1) is oscillatory on $\left[t_{0}, \infty\right)$, and $w^{2}(t)$ is a differentiable function such that

$$
\lim _{t \rightarrow \infty}\left[w^{2}\right]^{\prime}(t)=0
$$

then

$$
N\left(t_{0}, t\right) \sim \frac{1}{\pi} \int_{t_{0}}^{t} \frac{d s}{w^{2}(s)}, \quad t \rightarrow \infty,
$$

where the symbol $\sim$ means that the ratio of the two quantities tends to 1 as $t \rightarrow \infty$.

In this paper under some restrictions on the graininess of the time scale and the asymptotic behavior of the coefficient $w(t)$, we obtain an explicit Jeffreys, Wentzel, Kramers and Brillouin (JWKB) asymptotic representation of solutions of (1.1). Using this representa- 
tion we prove the analogue of Wiman's formula for $(1.1)$ on $\left[t_{0}, \infty\right)_{\mathbb{T}}$, which is given by

$$
N\left(t_{0}, t\right) \sim \frac{1}{\pi} \int_{t_{0}}^{t} \lim _{q \searrow \mu(s)} \frac{\tan ^{-1}\left(q w^{-2}(s)\right) \Delta s}{q}, \quad t \rightarrow \infty .
$$

First we recall some basic definitions and notation used in time scale analysis (see [2, $3])$. A time scale $\mathbb{T}$ is an arbitrary nonempty closed subset of the real numbers. Since we are interested in the asymptotic behavior of solutions of (1.1), we will consider time scales which are unbounded above, i.e., $\sup (\mathbb{T})=\infty$. For $t \in \mathbb{T}$ we define the forward and backward jump operators by

$$
\sigma(t)=\inf \{s \in \mathbb{T}, s>t\}, \quad \rho(t)=\sup \{s \in \mathbb{T}, s<t\} .
$$

The (forward) graininess function $\mu: \mathbb{T} \rightarrow[0, \infty)$ is defined by

$$
\mu(t)=\sigma(t)-t
$$

For $f: \mathbb{T} \rightarrow \mathbb{R}$ and $t \in \mathbb{T}$, we define the delta derivative $f^{\Delta}(t)$ to be the number (provided it exists) with the property that for any $\epsilon>0$, there exist a $\delta>0$ and a neighborhood $U=$ $(t-\delta, t+\delta) \cap \mathbb{T}$ of $t$ such that

$$
\left|f^{\sigma}(t)-f(s)-f^{\Delta}(t)(\sigma(t)-s)\right| \leq \epsilon|\sigma(t)-s|, \quad f^{\sigma}(t):=f(\sigma(t)),
$$

for all $s \in U$ (see [2]).

A function $f: \mathbb{T} \rightarrow \mathbb{R}$ is said to be $r d$-continuous provided it is continuous at right-dense points in $\mathbb{T}$ and at each left-dense point $t$ in $\mathbb{T}$ the left hand limit at $t$ exists (finite). The set of functions such that their $n$th delta derivative exists and is rd-continuous on $\mathbb{T}$ is denoted by $C_{r d}^{n}$. In (1.1) we assume that $w^{-2}(\cdot) \in C_{r d}$ and we say $u(\cdot)$ is a solution provided $u(\cdot) \in C_{r d}^{2}$ and $u^{\Delta \Delta}(t)+\left(w^{\sigma}(t)\right)^{-2} w^{-2}(t) u(t)=0$ for $t \in \mathbb{T}$. We say that a complex-valued function $f(\cdot)$ is regressive on $\mathbb{T}$ if $1+\mu(t) f(t) \neq 0$ for all $t \in \mathbb{T}$. The set of regressive functions on $C_{r d}$ will be denoted by $\mathcal{R}$. The set $\mathcal{R}$ along with the addition $\oplus$ defined by

$$
p \oplus q:=p+q+\mu p q
$$

forms an Abelian group called the regressive group ([2], p.58). If $p \in \mathcal{R}$ the (generalized) exponential function $e_{p}\left(t, t_{0}\right)$ is the unique solution of the IVP

$$
x^{\Delta}=p(t) x, \quad x\left(t_{0}\right)=1
$$

and is given by the formula

$$
e_{p}\left(t, t_{0}\right)=\exp \left[\int_{t_{0}}^{t} \lim _{q \searrow \mu(s)} \frac{1}{q} \ln (1+q p(s)) \Delta s\right]
$$

where $\ln$ is the principal logarithmic function. The set of regressive functions on $C_{r d}^{n}$ will be denoted by $\mathcal{R}^{n}$. 
A solution $u(\cdot)$ of (1.1) is said to have a zero at $a \in \mathbb{T}$ if $u(a)=0$, and it has a node at $\frac{a+\sigma(a)}{2}$ if $u(\sigma(a)) u(a)<0$. A generalized zero of $u(t)$ is defined as its zero or its node. A solution of (1.1) is said to be oscillatory if it has an infinite sequence of generalized zeros in $\mathbb{T}$, and nonoscillatory otherwise. Equation (1.1) is said to be oscillatory on $\mathbb{T}$ if all of its solutions are oscillatory, and nonoscillatory otherwise.

Wiman's result was extended by P. Hartman, Wintner [4], R. Potter [5], Z. Nehari [6], and H. Gingold [7]. Many different results on the oscillation of solutions of second order differential equations appear in the book of Swanson [8].

After the introduction of the time scale calculus by Hilger [3], oscillation theorems for second order dynamic equations on a time scale have been studied by many authors (see for example [9-11] and the references therein).

\section{Main results}

Since the basic method of this paper uses the asymptotic representation of solutions of (1.1) we will introduce some definitions and notation that will be important in what follows.

The following lemma will be important in the sequel. We will use the following notation:

$$
\Re[z]=\Re[x+i y]:=x, \quad \Im[z]=\Im[x+i y]=: y .
$$

Lemma 2.1 Assume $\eta:\left[t_{0}, \infty\right)_{\mathbb{T}} \rightarrow \mathbb{R}$ and $w:\left[t_{0}, \infty\right)_{\mathbb{T}} \rightarrow(0, \infty)$ are $r d$-continuous functions, $w(t)>0$ on $\left[t_{0}, \infty\right)_{\mathbb{T}}$ and let

$$
\theta_{1}(t):=\eta(t)+\frac{i}{w^{2}(t)}, \quad \theta_{2}(t):=\eta(t)-\frac{i}{w^{2}(t)}=\bar{\theta}_{1}(t)
$$

for $t \in\left[t_{0}, \infty\right)_{\mathbb{T}}$. Then $\Re\left[\theta_{1}\right], \Im\left[\theta_{1}\right], \Re\left[\theta_{2}\right], \Im\left[\theta_{2}\right] \in \mathcal{R}$, and

$$
e_{\theta_{1}}\left(t, t_{0}\right):=K\left(t, t_{0}\right) e_{\eta_{1}}\left(t, t_{0}\right) e^{i \Phi(t)}, \quad e_{\theta_{2}}\left(t, t_{0}\right):=K\left(t, t_{0}\right) e_{\bar{\eta}_{1}}\left(t, t_{0}\right) e^{-i \Phi(t)}
$$

where

$$
K\left(t, t_{0}\right)=\exp \left[\int_{t_{0}}^{t} \lim _{q \backslash \mu(s)} \frac{1}{2 q} \ln \left(1+\frac{q^{2}}{w^{4}(s)}\right) \Delta s\right],
$$

and

$$
\begin{aligned}
& \Phi(t)=\int_{t_{0}}^{t} \lim _{q \searrow \mu(s)} \frac{\Phi_{q}(s)}{q} \Delta s, \quad \eta_{1}(t)=\frac{\eta(t)}{1+i \frac{\mu(t)}{w^{2}(t)}}, \\
& \Phi_{q}(t)=\tan ^{-1}\left(\frac{q}{w^{2}(t)}\right) .
\end{aligned}
$$

Proof First note that if $\Im\left[\theta_{k}\right]=\frac{1}{w^{2}(t)} \neq 0$, and

$$
\left|1+\mu(t) \theta_{k}(t)\right|^{2}=\left(1+\mu(t) \Re\left[\theta_{k}(t)\right]\right)^{2}+\left(\mu(t) \Im\left[\theta_{k}(t)\right]\right)^{2}>0, \quad k=1,2,
$$


so $\theta_{k} \in \mathcal{R}, k=1,2$ and hence $e_{\theta_{k}}\left(t, t_{0}\right), k=1,2$ are well defined. Let $\eta_{1}$ be as in the statement of this lemma and consider

$$
\begin{aligned}
\eta_{1} \oplus \frac{i}{w^{2}} & =\eta_{1}+\frac{i}{w^{2}}+\mu \eta_{1} \frac{i}{w^{2}} \\
& =\eta_{1}\left[1+i \frac{\mu}{w^{2}}\right]+\frac{i}{w^{2}} \\
& =\eta+\frac{i}{w^{2}} \\
& =\theta_{1} .
\end{aligned}
$$

Hence

$$
e_{\theta_{1}}\left(t, t_{0}\right)=e_{\eta_{1} \oplus \frac{i}{w^{2}}}\left(t, t_{0}\right)=e_{\eta_{1}}\left(t, t_{0}\right) e_{\frac{i}{w^{2}}}\left(t, t_{0}\right) .
$$

In a similar manner one can show that

$$
\bar{\eta}_{1} \oplus \frac{-i}{w^{2}}=\theta_{2},
$$

and hence

$$
e_{\theta_{2}}\left(t, t_{0}\right)=e_{\bar{\eta}_{1} \oplus \frac{-i}{w^{2}}}\left(t, t_{0}\right)=e_{\bar{\eta}_{1}}\left(t, t_{0}\right) e_{\frac{-i}{w^{2}}}\left(t, t_{0}\right) .
$$

Using (1.5) we get

$$
\begin{aligned}
e_{\frac{i}{w^{2}}}\left(t, t_{0}\right) & =\exp \left[\int_{t_{0}}^{t} \lim _{q \searrow \mu(s)} \frac{1}{q} \ln \left(1+i \frac{q}{w^{2}(s)}\right) \Delta s\right] \\
& =e_{\frac{i}{w^{2}}}\left(t, t_{0}\right)=\exp \left[\int_{t_{0}}^{t} \lim _{q \searrow \mu(s)} \frac{1}{q}\left(\ln \sqrt{1+\frac{q^{2}}{w^{4}(s)}}+i \Phi_{q}(s)\right) \Delta s\right] .
\end{aligned}
$$

Hence

$$
\begin{aligned}
e_{\frac{i}{w^{2}}}\left(t, t_{0}\right) & =\exp \int_{t_{0}}^{t} \lim _{q \backslash \mu(s)}\left[\frac{1}{2 q} \ln \left(1+\frac{q^{2}}{w^{4}(s)}\right)+i \frac{\Phi_{q}(s)}{q}\right] \Delta s \\
& =K\left(t, t_{0}\right) e^{i \Phi(t)},
\end{aligned}
$$

where $K\left(t, t_{0}\right)$ and $\Phi(t)$ are given by (2.3) and (2.4). Similarly

$$
e_{\frac{-i}{w^{2}}}\left(t, t_{0}\right)=K\left(t, t_{0}\right) e^{-i \Phi(t)},
$$

and consequently the formulas (2.2) hold.

Example 2.1 Let $\theta_{k}, k=1,2$ be as in Lemma 2.1.

(1) If $\mathbb{T}=\left[t_{0}, \infty\right)$, then

$$
e_{\theta_{1}}\left(t, t_{0}\right)=e^{\int_{t_{0}}^{t} \eta_{1}(s) d s} e^{i \int_{t_{0}}^{t} \frac{d s}{w^{2}(s)}}, \quad e_{\theta_{2}}\left(t, t_{0}\right)=e^{\int_{t_{0}}^{t} \bar{\eta}_{1}(s) d s} e^{-i \int_{t_{0}}^{t} \frac{d s}{w^{2}(s)}} .
$$


(2) If $\mathbb{T}=\mathbb{N}$, then

$$
\begin{aligned}
& e_{\theta_{1}}(t, 1)=K(t, 1) e_{\eta_{1}}(t, 1) e^{i \sum_{k=1}^{t-1} \tan ^{-1} \frac{1}{w^{2}(k)}} \\
& e_{\theta_{2}}(t, 1)=K(t, 1) e_{\bar{\eta}_{1}}(t, 1) e^{-i \sum_{k=1}^{t-1} \tan ^{-1} \frac{1}{w^{2}(k)}}
\end{aligned}
$$

where

$$
K(t, 1)=\prod_{k=1}^{t-1} \ln \sqrt{1+\frac{1}{w^{4}(k)}}, \quad \eta_{1}(t)=\frac{\eta(t)}{1+i \frac{1}{w^{2}(t)}}
$$

Define $M: \mathbb{T} \rightarrow \mathbb{R}$ by

$$
M(t):=\left|p^{\Delta}+\frac{p^{2}}{w^{2}-p \mu+i \mu}\right|, \quad p(t)=-\frac{\left(w^{2}\right)^{\Delta}(t)}{2} .
$$

Note that the function $M(t)$ is defined from the JWKB approximation and may be chosen differently from another approximation (see Theorem 2.6).

Using the JWKB approximation method one can prove the following theorem.

Theorem 2.2 Assume that $w \in C_{r d}^{2}$ and the conditions

$$
\begin{aligned}
& \int_{t_{0}}^{\infty} M(s) \Delta s<\infty \\
& \mu(t) \lim _{q \searrow \mu(t)} \frac{\tan ^{-1}\left(q w^{-2}(t)\right)}{q} \leq \pi-\varepsilon, \quad t \in\left[t_{0}, \infty\right)_{\mathbb{T}}, \\
& \int_{t_{0}}^{\infty} \lim _{q \searrow \mu(s)} \frac{1}{q} \tan ^{-1}\left(\frac{p(s) q^{2}}{w^{4}(s)+q^{2}-p(s) w^{2}(s) q}\right) \Delta s=A<\infty
\end{aligned}
$$

are satisfied for some $\varepsilon>0$. Then (1.1) is oscillatory if and only if

$$
\int_{t_{0}}^{\infty} \lim _{q \searrow \mu(s)} \frac{\tan ^{-1}\left(q w^{-2}(s)\right) \Delta s}{q}=\infty
$$

Theorem 2.3 Assume that $w \in C_{r d}^{2}$ and conditions (2.6)-(2.8) are satisfied. Then

$$
\left|N\left(t_{0}, t\right)+\frac{1}{2}-\frac{1}{\pi} \int_{t_{0}}^{t} \lim _{q \searrow \mu(s)} \frac{\tan ^{-1}\left(q w^{-2}(s)\right) \Delta s}{q}\right| \leq 1 .
$$

Corollary 2.4 Assume that $w \in C_{r d}^{2}$ and conditions (2.6)-(2.8) are satisfied. Then

$$
N\left(t_{0}, t\right) \sim \frac{1}{\pi} \int_{t_{0}}^{t} \lim _{q \searrow \mu(s)} \frac{\tan ^{-1}\left(q w^{-2}(s)\right) \Delta s}{q}, \quad t \rightarrow \infty .
$$

Example 2.2 Consider the difference equation

$$
\Delta^{2} u_{n}+\frac{u_{n}}{\sqrt{n(n+1)}}=0
$$


on the discrete time scale $\mathbb{T}=\mathbb{Z}$. We have

$$
\begin{aligned}
& w(n)=w_{n}=n^{1 / 4}, \\
& p=-\frac{1}{2}\left(w^{2}\right)^{\Delta}=-\frac{1}{2}\left(n^{1 / 2}\right)^{\Delta}=\frac{n^{1 / 2}-(n+1)^{1 / 2}}{2}, \quad M(n) \sim n^{-3 / 2}, \quad n \rightarrow \infty .
\end{aligned}
$$

Conditions (2.6)-(2.8) are satisfied for sufficiently large $n$, and from (2.10) we get

$$
N(n) \sim \frac{1}{\pi} \sum_{k=1}^{n-1} \tan ^{-1}\left(\frac{1}{\sqrt{k}}\right), \quad n \rightarrow \infty .
$$

For a continuous time scale $(\mu(t) \equiv 0)$ conditions (2.7)-(2.8) are automatically satisfied, and from Lemma 2.1, Theorem 2.2 we get the following corollary.

Corollary 2.5 Assume that $w(t)$ is twice differentiable on $\left[t_{0}, \infty\right)$ and the condition

$$
\int_{t}^{\infty}\left|w(t) w^{\prime \prime}(t)\right| d t<\infty, \quad t \geq t_{0}
$$

is satisfied. Then (1.3) is true, and (1.1) is oscillatory if and only if

$$
\int_{t_{0}}^{\infty} \frac{d s}{w^{2}(s)}=\infty
$$

Note that the necessary part of Corollary 2.5 is due to Leighton [12] under the assumption that $w$ is a monotone function.

For monotonic functions $w$, Wiman's condition (1.2) is less restrictive than (2.11) (for example $\left.w^{2}(t)=t \ln ^{-\varepsilon}(t), \varepsilon>0\right)$. His proof is based on the transformation of the time variable which causes problems in the time scale setting. In this paper we give a new proof of Wiman's formula that does not require the monotonicity of $w$, and it is based on an asymptotic representation of solutions of (1.1).

Example 2.3 From Corollary 2.5 it follows that the equation

$$
u^{\prime \prime}(t)+\frac{a}{t^{2 \gamma}} u(t)=0, \quad a>0,
$$

is oscillatory if and only if $\gamma<1$. Indeed from $\gamma<1$ it follows that both conditions (2.11) and (2.12) are satisfied. Condition (2.11) is restrictive, but without it Corollary 2.5 is not true since for the nonoscillatory equation $u^{\prime \prime}+\frac{1}{4 t^{2}} u=0$ condition (2.12) is satisfied. It is well known (Kneser [13]) that (2.13) is nonoscillatory if $a t^{-2 \gamma} \leq \frac{1}{4} t^{-2}$, and oscillatory if $a t^{-2 \gamma}>$ $\frac{1}{4} t^{-2}$. Our condition $\gamma<1$ is stronger, but it provides explicit asymptotic representations of the solutions and their derivatives as well.

Example 2.4 For the example

$$
u^{\prime \prime}(t)+\left(\frac{a \ln ^{2 \beta}(t)}{t^{2}}+\frac{\sin (t)}{t^{3}}\right) u(t)=0, \quad a>0
$$


condition (2.11) is satisfied if $\beta>1$, and condition (2.12) is satisfied if $\beta>-1$. For this example Leighton's necessary criterion for oscillation [12] does not apply since $w^{-4}(t)=$ $a \ln ^{2 \beta}(t) t^{-2}+\sin (t) t^{-3}$ is not monotone.

Using the Hartman-Wintner [4] approximation one can prove the following theorem.

Theorem 2.6 Assume that $w \in C_{r d}^{2}$ and for some $\varepsilon_{1}, \varepsilon \in(0,1)$ the conditions

$$
\begin{aligned}
& 1-p^{2}(t) \geq \varepsilon, \quad p(t):=-\frac{1}{2}\left(w^{2}\right)^{\Delta}(t), \quad t \in\left[t_{0}, \infty\right)_{\mathbb{T}}, \\
& \int_{t}^{\infty} \frac{\left|r^{\Delta}(s)\right| \Delta s}{\sqrt{1-\left|p^{\sigma}(s)\right|^{2}}}<\infty, \quad r(t)=p(t)+i-i \sqrt{1-p^{2}(t)}, \\
& \mu(t) \lim _{q \searrow \mu(t)} \frac{\tan ^{-1}\left(q w^{-2}(t) \sqrt{1-p^{2}(t)}\right)}{q} \leq \pi-\varepsilon_{1}, \quad t \in\left[t_{0}, \infty\right)_{\mathbb{T}}, \\
& \int_{t_{0}}^{\infty} \lim _{q \searrow \mu(s)} \frac{1}{q} \tan ^{-1}\left(\frac{q p(s) \sqrt{1-p^{2}(s)}}{w^{2}(s)\left(w^{2}(s)-p(s) q\right)+q^{2}\left(1-p^{2}(s)\right)}\right) \Delta s=A<\infty
\end{aligned}
$$

are satisfied.

Then (1.1) is oscillatory if and only if

$$
\int_{t_{0}}^{\infty} \lim _{q \searrow \mu(s)} \frac{\tan ^{-1}\left(q w^{-2}(s) \sqrt{1-p^{2}(s)}\right)}{q} \Delta s=\infty .
$$

Theorem 2.7 Assume that $w \in C_{r d}^{2}$ and conditions (2.15)-(2.18) are satisfied. Then Neharis generalization of Wiman's formula (see [6]) is true:

$$
N\left(t_{0}, t\right) \sim \frac{1}{\pi} \int_{t_{0}}^{t} \lim _{q \searrow \mu(s)} \frac{\tan ^{-1}\left(q w^{-2}(s) \sqrt{1-p^{2}(s)}\right) \Delta s}{q}, \quad t \rightarrow \infty .
$$

\section{Proofs}

To get the asymptotic representation of solutions of the equation

$$
L[u(t)]=u^{\Delta \Delta}(t)+\frac{u(t)}{\left(w^{\sigma} w(t)\right)^{2}}=0, \quad t \in\left[t_{0}, \infty\right)_{\mathbb{T}}
$$

we will use the following theorem.

Theorem 3.1 ([14], Theorem 2.4) Let $u_{1}, u_{2} \in C_{r d}^{2}$ be complex-valued functions such that

$$
\begin{aligned}
& W\left[u_{1}, u_{2}\right]:=u_{1}(t) u_{2}^{\Delta}(t)-u_{1}^{\Delta}(t) u_{2} \neq 0, \quad t \in\left[t_{0}, \infty\right)_{\mathbb{T}} \\
& \int_{t_{0}}^{\infty} M_{0}(t) \Delta t<\infty, \quad m, n=1,2
\end{aligned}
$$

where

$$
M_{0}(t)=\max _{m, n=1,2}\left|H_{m n}(t)\right|, \quad H_{m n}(t):=\frac{u_{m}^{\sigma}(t) L\left[u_{n}(t)\right]}{W^{\sigma}\left[u_{1}, u_{2}\right]},
$$




$$
L[u(t)]=u^{\Delta \Delta}(t)+P(t) u^{\Delta}(t)+Q(t) u(t) .
$$

Then for arbitrary constants $C_{1}, C_{2}$ there exists a solution $u$ of (3.1) that can be written in the form

$$
\begin{aligned}
& u(t)=\left[C_{1}+\delta_{1}(t)\right] u_{1}(t)+\left[C_{2}+\delta_{2}(t)\right] u_{2}(t), \\
& u^{\Delta}(t)=\left[C_{1}+\delta_{1}(t)\right] u_{1}^{\Delta}(t)+\left[C_{2}+\delta_{2}(t)\right] u_{2}^{\Delta}(t),
\end{aligned}
$$

where the error vector-function $\delta(t)$ satisfies

$$
\|\delta(t)\| \leq\|C\|\left(-1+\exp \left\{\int_{t}^{\infty} M_{0}(s) \Delta s\right\}\right)
$$

where $M_{0}(t)$ is defined as in (3.4), $\|\delta\|$ is the Euclidean vector (or matrix) norm: $\|\delta(t)\|=$ $\sqrt{\sum_{k=1}^{2} \delta_{k}^{2}(t)}$, and $\delta_{k}(t), k=1,2$ are the entries of the vector $\delta(t)$.

Remark 3.1 If we seek asymptotic solutions $u_{n}$ of (3.1) in the Euler form,

$$
u_{n}(t)=e_{n}(t)=e_{\theta_{n}}\left(t, t_{0}\right), \quad n=1,2,
$$

then in view of $L\left[e_{n}(t)\right]=R\left(\theta_{n}(t)\right) e_{n}$, we see that the formula (3.4) becomes

$$
M_{0}(s)=\max _{m, n=1,2}\left|\frac{e_{n}(s) R\left(\theta_{n}(s)\right)}{e_{m}^{\sigma}(s)\left[\theta_{1}^{\sigma}(s)-\theta_{2}^{\sigma}(s)\right]}\right|
$$

where the Riccati functions $R\left(\theta_{n}(t)\right), n=1,2$ are defined by

$$
R\left(\theta_{n}(t)\right)=\theta_{n}^{\Delta}(t)+\theta_{n}^{\sigma}(t) \theta_{n}(t)+\frac{1}{\left(w^{\sigma}(t) w(t)\right)^{2}}, \quad n=1,2 .
$$

Theorem 3.2 Assume $w \in C_{r d}^{2}$, and condition (3.3) is satisfied with

$$
M_{0}(s)=\frac{1}{2}\left|p^{\Delta}(s)+\frac{p^{2}(s)}{w^{2}(s)-p \mu(s)+i \mu(s)}\right|, \quad p(t)=-\frac{\left(w^{2}\right)^{\Delta}(t)}{2} .
$$

Then for arbitrary constants $C_{1}, C_{2}$ there exists a solution $u$ of (3.1) that can be written in the form (3.6) and (3.7), with error estimate given by (3.8).

Proof In Lemma 2.1, take $\eta(t)=\frac{-p(t)}{w^{2}(t)}$, where $p(t)$ will be chosen later in this proof. Then

$$
\theta_{1}(t)=\frac{i-p(t)}{w^{2}(t)}, \quad \theta_{2}(t)=\frac{-i-p(t)}{w^{2}(t)}
$$

are regressive functions. Note that

$$
\begin{aligned}
W\left[e_{\theta_{1}}\left(t, t_{0}\right), e_{\theta_{2}}\left(t, t_{0}\right)\right] & =\left(\theta_{2}(t)-\theta_{1}(t)\right) e_{\theta_{1}}\left(t, t_{0}\right) e_{\theta_{2}}\left(t, t_{0}\right) \\
& =-\frac{2 i}{w^{2}} e_{\theta_{1} \oplus \theta_{2}}\left(t, t_{0}\right) \neq 0,
\end{aligned}
$$


so condition (3.2) of Theorem 3.1 is satisfied, where $u_{i}(t)=e_{\theta_{i}}\left(t, t_{0}\right), i=1,2$.

By the quotient rule

$$
\begin{aligned}
\theta_{1}^{\Delta} & =\frac{(i-p)^{\Delta} w^{2}-(i-p)\left(w^{2}\right)^{\Delta}}{\left(w^{\sigma} w\right)^{2}} \\
& =-\frac{p^{\Delta}}{\left(w^{\sigma}\right)^{2}}+\frac{(i-p) 2 k}{\left(w^{\sigma} w\right)^{2}}
\end{aligned}
$$

where $k=-\frac{\left(w^{2}\right)^{\Delta}}{2}$. Then

$$
\begin{aligned}
R\left(\theta_{1}\right) & =\frac{-p^{\Delta} w^{2}+(i-p) 2 k+(i-p)\left(i-p^{\sigma}\right)+1}{\left(w^{\sigma} w\right)^{2}} \\
& =\frac{p^{2}-2 p(k+i)+2 i k-p^{\Delta}\left(w^{2}+i \mu-p \mu\right)}{\left(w^{\sigma} w\right)^{2}}
\end{aligned}
$$

where we used $p^{\sigma}=p+\mu p^{\Delta}$. Using

$$
1+\mu \theta_{1}=\left(w^{2}+i \mu-p \mu\right) / w^{2} \text { and }\left(\theta_{1}-\theta_{2}\right)^{\sigma}=2 i\left(w^{\sigma}\right)^{-2}
$$

we get

$$
\frac{2 i R\left(\theta_{1}\right)}{\left(1+\mu \theta_{1}\right)\left(\theta_{1}-\theta_{2}\right)^{\sigma}}=-p^{\Delta}+\frac{p^{2}-2 p(k+i)+2 i k}{w^{2}+i \mu-p \mu} .
$$

Choosing the JWKB approximation:

$$
p(t)=k(t):=-\frac{\left(w^{2}\right)^{\Delta}(t)}{2}
$$

we get

$$
2 M_{0}(s)=\max _{m, n=1,2}\left|\frac{2 i R_{n}(s)}{\left(1+\mu \theta_{m}(s)\right)\left[\theta_{1}^{\sigma}(s)-\theta_{2}^{\sigma}(s)\right]}\right|=\left|-p^{\Delta}-\frac{p^{2}}{w^{2}+i \mu-p \mu}\right| .
$$

Note that there is another possible choice of $p(t)$ as a solution of the quadratic equation $p^{2}-2 p(k+i)+2 i k=0$ (the Hartman-Wintner approximation, see [4]).

Proof of Theorem 2.2 Let $\theta_{1}$ and $\theta_{2}$ be defined as in the proof of Theorem 3.2. Then by Lemma 2.1 with $\eta(t)=-\frac{p(t)}{w^{2}(t)}$

$$
e_{\theta_{1}}\left(t, t_{0}\right)=K\left(t, t_{0}\right) e_{\eta_{1}}\left(t, t_{0}\right) e^{i \Phi(t)}, \quad e_{\theta_{2}}\left(t, t_{0}\right)=K\left(t, t_{0}\right) e_{\bar{\eta}_{1}}\left(t, t_{0}\right) e^{-i \Phi(t)},
$$

where $K\left(t, t_{0}\right)$ and $\Phi(t)$ are given by (2.3) and (2.4).

Using the Euler formula we have

$$
\begin{aligned}
& u_{1}(t)=e_{\theta_{1}}\left(t, t_{0}\right)=K\left(t, t_{0}\right)(\cos (\Phi(t))+i \sin (\Phi(t))) e_{\eta_{1}}\left(t, t_{0}\right), \\
& u_{2}(t)=e_{\theta_{2}}\left(t, t_{0}\right)=K\left(t, t_{0}(\cos (\Phi(t))-i \sin (\Phi(t))) e_{\bar{\eta}_{1}}\left(t, t_{0}\right) .\right.
\end{aligned}
$$


From Theorem 3.2, a general solution of (3.1) is of the form

$$
\begin{aligned}
u(t) & =\left(C_{1}+\delta_{1}(t)\right) e_{\theta_{1}}\left(t, t_{0}\right)+\left(C_{2}+\delta_{2}(t)\right) e_{\theta_{2}}\left(t, t_{0}\right) \\
& =N_{1}(t) \cos (\Phi(t))+N_{2}(t) \sin (\Phi(t))
\end{aligned}
$$

where

$$
\begin{aligned}
& N_{1}(t)=K\left(t, t_{0}\right)\left[\left(\left(C_{1}+\delta_{1}(t)\right) e_{\eta_{1}}(t)+\left(C_{2}+\delta_{2}(t)\right) e_{\bar{\eta}_{1}}(t)\right)\right], \\
& N_{2}(t)=i K\left(t, t_{0}\right)\left[\left(\left(C_{1}+\delta_{1}(t)\right) e_{\eta_{1}}(t)-\left(C_{2}+\delta_{2}(t)\right) e_{\bar{\eta}_{1}}(t)\right)\right] .
\end{aligned}
$$

Assume $u(t)$ is a real-valued solution of (1.1) and $C_{1}, C_{2}$ be arbitrary real constants. We will prove that $N_{1}(t), N_{2}(t)$ are also real-valued functions.

Indeed by solving the system (3.6) and (3.7) for $C_{j}+\delta_{j}$ we get

$$
C_{1}+\delta_{1}=\frac{u_{2}^{\Delta} u-u^{\Delta} u_{2}}{u_{1} u_{2}^{\Delta}-u_{2} u_{1}^{\Delta}}, \quad C_{2}+\delta_{2}=\frac{u^{\Delta} u_{1}-u_{1}^{\Delta} u}{u_{1} u_{2}^{\Delta}-u_{2} u_{1}^{\Delta}}
$$

which implies $\overline{C_{1}+\delta_{1}}=C_{2}+\delta_{2}$, which in turn implies that $\delta_{1}(t), \delta_{2}(t)$ are complex conjugates of each other. Then, since $e_{\eta_{1}}, e_{\bar{\eta}_{1}}$ are complex conjugates, from (3.18) it follows that $N_{1,2}$ are real-valued functions.

Define $\Lambda(t)$ so that

$$
\sin (\Lambda(t))=\frac{N_{1}(t)}{\sqrt{N_{1}^{2}(t)+N_{2}^{2}(t)}}=\frac{\left(C_{1}+\delta_{1}(t)\right) e_{\eta_{1}}\left(t, t_{0}\right)+\left(C_{2}+\delta_{2}(t)\right) e_{\bar{\eta}_{1}}\left(t, t_{0}\right)}{2\left|e_{\eta_{1}}\left(t, t_{0}\right)\right| \sqrt{\left[C_{1}+\delta_{1}(t)\right]\left[C_{2}+\delta_{2}(t)\right]}}
$$

then from (3.17) we get

$$
u(t)=\sqrt{N_{1}^{2}+N_{2}^{2}} \sin (\Phi(t)+\Lambda(t))
$$

We extend the domain of the function

$$
\Phi(t)=\int_{t_{0}}^{t} \lim _{q \searrow \mu(s)} \frac{\Phi_{q}(s)}{q} \Delta s, \quad t \in\left[t_{0}, \infty\right)_{\mathbb{T}}
$$

for $t$ in the real interval $\left(t_{1}, \sigma\left(t_{1}\right)\right)$, when $\sigma\left(t_{1}\right) \neq t_{1}$ by the formula

$$
\Phi_{e}(t)=\frac{\left(\sigma\left(t_{1}\right)-t\right) \Phi\left(t_{1}\right)+\left(t-t_{1}\right) \Phi\left(\sigma\left(t_{1}\right)\right)}{\sigma\left(t_{1}\right)-t_{1}}, \quad t \in\left(t_{1}, \sigma\left(t_{1}\right)\right)
$$

Note that $\Phi_{e}(t)$ is linear and continuous on $\left(t_{1}, \sigma\left(t_{1}\right)\right)$ and

$$
\Phi_{e}\left(t_{1}\right)=\Phi\left(t_{1}\right), \quad \Phi_{e}\left(\sigma\left(t_{1}\right)\right)=\Phi\left(\sigma\left(t_{1}\right)\right)
$$

In the same way one can extend the function $\Phi$ with domain $\left[t_{0}, \infty\right)_{\mathbb{T}}$ to $\Phi_{e}$ with domain the real interval $\left[t_{0}, \infty\right)$. Since $\Phi(t) \in C_{r d}^{1}\left(\left(t_{0}, \infty\right)_{\mathbb{T}}\right)$ the extended function $\Phi_{e} \in C\left(t_{0}, \infty\right)$.

Later we will show that there exist points $z_{n} \in\left(t_{0}, \infty\right)$ (which may not belong to $\left[t_{0}, \infty\right)_{\mathbb{T}}$ ) such that

$$
\frac{\Phi_{e}\left(z_{n}\right)}{n}+\frac{\Lambda_{e}\left(z_{n}\right)}{n}=\pi, \quad n=0, \pm 1, \pm 2, \pm 3, \ldots,
$$


so the zeros of the extended solution $u(t)$ are located at $z_{n}$. Assuming $z_{1}>t_{0}$ from (2.4) we get

$$
\begin{aligned}
& \Phi(t)=\int_{t_{0}}^{t} \lim _{q \searrow \mu(s)} \frac{\tan ^{-1}\left(q w^{-2}(s)\right) \Delta s}{q}, \quad t \in\left[t_{0}, \infty\right)_{\mathbb{T}}, \\
& \Lambda(t)=\sin ^{-1}\left(\frac{N_{1}(t)}{\sqrt{N_{1}^{2}(t)+N_{2}^{2}(t)}}\right) \leq \frac{\pi}{2}, \quad t \in\left[t_{0}, \infty\right)_{\mathbb{T}} .
\end{aligned}
$$

Further we have

$$
e_{\eta_{1}}\left(t, t_{0}\right)=\exp \left\{\int_{t_{0}}^{t} \frac{\ln \left|1+\mu(s) \eta_{1}(s)\right| \Delta s}{\mu(s)}\right\} \exp \left\{\int_{t_{0}}^{t} \frac{i \beta(s) \Delta s}{\mu(s)}\right\},
$$

where

$$
\beta(s):=\operatorname{Arg}\left(1+\mu \eta_{1}(s)\right) .
$$

From

$$
\begin{aligned}
& 1+\mu \eta_{1}=1-\frac{p \mu}{w^{2}+i \mu}=1-\frac{p \mu\left(w^{2}-i \mu\right)}{w^{4}+\mu^{2}}, \\
& \left|1+\mu \eta_{1}\right|^{2}=\left(1-\frac{p \mu w^{2}}{w^{4}+\mu^{2}}\right)^{2}+\frac{p^{2} \mu^{4}}{\left(w^{4}+\mu^{2}\right)^{2}}>0,
\end{aligned}
$$

and so $e_{\eta_{1}}\left(t, t_{0}\right)$ is well defined. Indeed (3.25) is true when $\mu(t)=0$ and when $\mu(t) \neq 0$.

Further

$$
1+\mu \eta_{1}=\left|1+\mu \eta_{1}\right| e^{i \beta}, \quad \tan (\beta)=\frac{p \mu^{2}}{w^{4}+\mu^{2}-p \mu w^{2}} .
$$

From (2.8) and (3.19) we see that the following limits exist:

$$
\begin{aligned}
& \lim _{t \rightarrow \infty} \frac{e_{\eta_{1}}(t)}{e_{\bar{\eta}_{1}}(t)}=\exp \int_{t_{0}}^{\infty} \frac{2 i \beta(s) \Delta s}{\mu(s)}=A_{1}, \\
& \lim _{t \rightarrow \infty} \sin (\Lambda(t))=A_{2} .
\end{aligned}
$$

Further, since $\sin ^{-1}(x)$ is a continuous function on its domain, we get

$$
\lim _{z_{n} \rightarrow \infty} \frac{\Lambda\left(z_{n}\right)}{n}=0
$$

and from (3.21)

$$
\frac{1}{\pi}\left(\Phi\left(z_{n}\right)+o(1)\right)=n .
$$

Note that from the Leibniz formula (see Theorem 1.117 [2])

$$
\left(\int_{t_{0}}^{t} f(t, s) \Delta s\right)^{\Delta}(t)=\int_{t_{0}}^{t} f^{\Delta t}(t, s) \Delta s+f(\sigma(t), t)
$$


we get

$$
\Phi_{e}^{\Delta}\left(z_{n}\right)=\lim _{q \searrow \mu\left(\sigma\left(z_{n}\right)\right)} \frac{\tan ^{-1}\left(q w^{-2}\left(\sigma\left(z_{n}\right)\right)\right)}{q}>0,
$$

which means that $\Phi_{e}\left(z_{n}\right)$ is continuous and increasing (see Theorem 1.76 [2]), and hence it is an invertible function on $\left(t_{0}, \infty\right)$, and $z_{n} \in\left(t_{0}, \infty\right)$ exists for each $n \geq n_{0}$.

To show that the solution $u(t)$ of (3.1) has infinitely many generalized zeros on the time scale $\mathbb{T}$ we will prove that between two zeros on $\mathbb{R}$ of the solution there exists a generalized zero of $u(t)$ in $\mathbb{T}$.

We will show that for all $n \geq n_{0}$, for some $n_{0}>0$, there exists a point $\tau_{m} \in \mathbb{T}$ between two zeros of $u(t) z_{n}, z_{n+1} \in \mathbb{R}$ :

$$
z_{n}<\tau_{m} \leq z_{n+1}, \quad n \geq n_{0} .
$$

We prove this by contradiction assuming that there is no such point $\tau_{m} \in \mathbb{T}$. That is,

$$
\tau_{m-1} \leq z_{n}<z_{n+1}<\tau_{m}, \quad \tau_{m}=\sigma\left(\tau_{m-1}\right), \quad n \geq n_{0} .
$$

From (3.28) we get

$$
\Phi\left(z_{n+1}\right)-\Phi\left(z_{n}\right)=\pi-o(1), \quad n \rightarrow \infty,
$$

or

$$
\int_{z_{n}}^{z_{n+1}} \lim _{q \searrow \mu} \frac{\tan ^{-1}\left(q w^{-2}(s)\right)}{q} \Delta s=\pi-o(1), \quad z_{n} \rightarrow \infty .
$$

Further we have the estimate

$$
\int_{\tau_{m-1}}^{\tau_{m}} \lim _{q \searrow \mu} \frac{\tan ^{-1}\left(q w^{-2}(s)\right)}{q} \Delta s \geq \int_{z_{n}}^{z_{n+1}} \lim _{q \searrow \mu} \frac{\tan ^{-1}\left(q w^{-2}(s)\right)}{q} \Delta s=\pi-o(1),
$$

or, since $\tau_{m}=\sigma\left(\tau_{m-1}\right)$,

$$
\tan ^{-1}\left[\mu\left(\tau_{m-1}\right) w^{-2}\left(\tau_{m-1}\right)\right] \geq \pi-o(1), \quad \tau_{m}>z_{n} \rightarrow \infty
$$

The last estimate contradicts (2.7) for sufficiently large $n$.

Further from (3.29)

$$
2 n \pi(1-o(1))=\Phi\left(z_{2 n}\right)<\Phi\left(\tau_{m}\right) \leq \Phi\left(z_{2 n+1}\right)=(2 n+1) \pi(1-o(1)),
$$

and similarly

$$
(2 n-1) \pi(1-o(1))=\Phi\left(z_{2 n-1}\right)<\Phi\left(\tau_{m-1}\right) \leq \Phi\left(z_{2 n}\right)=2 n \pi(1-o(1)),
$$

So

$$
\sin \left(\Phi\left(\tau_{m}\right)\right)>0, \quad \sin \left(\Phi\left(\tau_{m-1}\right)\right)<0,
$$


which means that the point $\tau_{m-1}$ is the generalized zero. So (3.1) is oscillatory if and only if (2.9) is satisfied.

Proof of Theorem 2.3 Assuming $z_{1}>t_{0}$ the number $N\left(t, t_{0}\right)=n$ of generalized zeros of (3.1) on $\left(t_{0}, z_{n}\right)$ is given by (3.28). From (3.21) and (3.22)

$$
\left|\frac{\Phi_{e}\left(z_{n}\right)}{\pi}-n\right|=\left|-\frac{\Lambda_{e}\left(z_{n}\right)}{\pi}\right| \leq \frac{1}{2}
$$

or

$$
\left|\int_{t_{0}}^{z_{n}} \lim _{q \searrow \mu(s)} \frac{\tan ^{-1}\left(q w^{-2}(s)\right)}{\pi q} \Delta s-n\right| \leq \frac{1}{2}, \quad\left|\int_{t_{0}}^{z_{n+1}} \frac{\tan ^{-1}\left(\mu w^{-2}(s)\right) \Delta s}{\pi \mu(s)}-n-1\right| \leq \frac{1}{2} .
$$

Since between two zeros $z_{n}, z_{n+1}$ there is a generalized zero $t \in \mathbb{T}$, that is, $z_{n} \leq t<z_{n+1}$, we get

$$
\begin{aligned}
& \int_{t_{0}}^{t} \frac{\tan ^{-1}\left(\mu w^{-2}(s)\right) \Delta s}{\pi \mu(s)} \leq \int_{t_{0}}^{z_{n+1}} \frac{\tan ^{-1}\left(\mu w^{-2}(s)\right) \Delta s}{\pi \mu(s)} \leq n+1+\frac{1}{2}, \\
& \int_{t_{0}}^{t} \frac{\tan ^{-1}\left(\mu w^{-2}(s)\right) \Delta s}{\pi \mu(s)} \geq \int_{t_{0}}^{z_{n}} \frac{\tan ^{-1}\left(\mu w^{-2}(s)\right) \Delta s}{\pi \mu(s)} \geq n-1+\frac{1}{2}, \\
& \left|N(t)+\frac{1}{2}-\int_{t_{0}}^{t} \frac{\tan ^{-1}\left(\mu(s) w^{-2}(s)\right) \Delta s}{\pi \mu(s)}\right| \leq 1, \\
& N(t)=\left[\int_{t_{0}}^{t} \frac{\tan ^{-1}\left(\mu(s) w^{-2}(s)\right) \Delta s}{\pi \mu(s)}-\frac{1}{2}\right] \pm 1
\end{aligned}
$$

when $[\alpha]$ is the integral part of $\alpha \in \mathbb{R}$.

Proof of Corollary 2.5 To deduce Corollary 2.5 from Lemma 2.1, Theorem 2.2 note that for the continuous case $\mu \equiv 0$ and conditions (2.7) and (2.8) are automatically satisfied. From (2.5) we get

$$
M(t)=\left|w^{\prime \prime}(t) w(t)\right|,
$$

so condition (2.6) simplifies to (2.11), and (2.9) becomes (2.12).

Proofs of Theorem 2.6 and Theorem 2.7 The proofs of Theorem 2.6 and Theorem 2.7 are similar to the proofs of Theorem 2.2 and Theorem 2.3 correspondingly. The only difference is a different choice in (2.1) of the functions

$$
\eta=\frac{-r}{w^{2}}, \quad r=p+i-i \sqrt{1-p^{2}}, p=-\frac{\left(w^{2}\right)^{\Delta}}{2}
$$

where $r(t)$ is a solution of quadratic equation $r^{2}-2 r(i+p)+2 i p=0$. By this choice we have

$$
\theta_{1}=\eta+\frac{i}{w^{2}}=\frac{i-r}{w^{2}}=\frac{i \sqrt{1-p^{2}}-p}{w^{2}}, \quad \theta_{2}=\overline{\theta_{1}}=-\frac{p+i \sqrt{1-p^{2}}}{w^{2}}
$$


and by a few different calculations, using (3.10) and (3.11), we get

$$
\begin{aligned}
& \theta_{1}-\theta_{2}=\frac{2 i \sqrt{1-p^{2}}}{w^{2}}, \quad \Re\left[\theta_{1}\right]=\frac{-p}{w^{2}}, \quad \Im\left[\theta_{1}\right]=\frac{\sqrt{1-p^{2}}}{w^{2}}, \\
& \frac{2 i R\left(\theta_{1}\right)}{\left(1+\mu \theta_{1}\right)\left(\theta_{1}-\theta_{2}\right)^{\sigma}}=\frac{-1}{\sqrt{1-\left(p^{\sigma}\right)^{2}}}\left(r^{\Delta}+\frac{r^{2}-2 r(p+i)+2 i p}{w^{2}+i \mu-r \mu}\right)=\frac{-r^{\Delta}}{\sqrt{1-\left(p^{\sigma}\right)^{2}}} .
\end{aligned}
$$

For $t \in\left[t_{0}, \infty\right)_{\mathbb{T}}$ we have

$$
\begin{aligned}
& e_{\theta_{1}}\left(t, t_{0}\right)=e_{\Re\left[\theta_{1}\right]+i \Im\left[\theta_{1}\right]}\left(t, t_{0}\right)=K\left(t, t_{0}\right) e_{\eta_{2}}\left(t, t_{0}\right) e^{i \Psi(t)}, \\
& e_{\theta_{2}}\left(t, t_{0}\right):=K\left(t, t_{0}\right) e_{\eta_{2}}\left(t, t_{0}\right) e^{-i \Psi(t)},
\end{aligned}
$$

where

$$
\begin{aligned}
& \eta_{2}=\frac{\Re\left[\theta_{1}\right]}{1+i \mu \Im\left[\theta_{1}\right]}, \quad K\left(t, t_{0}\right)=\exp \left[\int_{t_{0}}^{t} \lim _{q \unlhd \mu(s)} \frac{\ln \left(1+\left(\Im\left[\theta_{1}\right]\right)^{2}\right)}{2 q} \Delta s\right], \\
& \Psi(t)=\int_{t_{0}}^{t} \lim _{q \searrow \mu(s)} \frac{\Psi_{q}(s)}{q} \Delta s, \quad \Psi_{q}=\tan ^{-1}\left(q \Im\left[\theta_{1}\right]\right) .
\end{aligned}
$$

In view of (2.15) in the case $\mu(t)>0$ we have $\mu(t) \Im\left[\theta_{1}(t)\right]=\frac{\mu(t) \sqrt{1-p^{2}(t)}}{w^{2}(t)} \neq 0$, and

$$
\left|1+\mu \theta_{k}\right|^{2}=\left(1+\mu \Re\left[\theta_{k}\right]\right)^{2}+\left(\mu \Im\left[\theta_{k}\right]\right)^{2}>0,
$$

so $\theta_{k} \in \mathcal{R}, k=1,2$, and hence $e_{\theta_{k}}\left(t, t_{0}\right), k=1,2$ are well defined.

In view of the choice (3.32) we have

$$
\begin{aligned}
& \eta_{2}=\frac{-p}{w^{2}+i \mu \sqrt{1-p^{2}}}, \\
& 1+\mu \eta_{2}=1-\frac{p \mu}{w^{2}+i \mu \sqrt{1-p^{2}}}=1-\frac{p \mu\left(w^{2}-i \mu \sqrt{1-p^{2}}\right)}{w^{4}+\mu^{2}\left(1-p^{2}\right)}, \\
& \left|1+\mu \eta_{2}\right|^{2}=\left(1-\frac{p \mu w^{2}}{w^{4}+\mu^{2}\left(1-p^{2}\right)}\right)^{2}+\frac{p^{2} \mu^{4}\left(1-p^{2}\right)}{\left(w^{4}+\mu^{2}\left(1-p^{2}\right)\right)^{2}}>0
\end{aligned}
$$

in view of (2.15). So $e_{\eta_{2}}$ is well defined. Also

$$
1+\mu \eta_{2}=\left|1+\mu \eta_{2}\right| e^{i \beta}, \quad \tan (\beta)=\frac{\mu p \sqrt{1-p^{2}}}{w^{2}\left(w^{2}-p \mu\right)+\mu^{2}\left(1-p^{2}\right)} .
$$

The rest of the proof is similar to the earlier proof. 


\section{Author details}

'Department of Mathematics, University of Nebraska-Lincoln, Lincoln, NE 68588-0130, USA. ${ }^{2}$ Department of Mathematics, Kent State University at Stark, 6000 Frank Ave. NW, Canton, OH 44720-7599, USA.

\section{Acknowledgements}

The authors would like to thank anonymous reviewers for very useful and constructive comments that helped to improve the original manuscript.

\section{Received: 8 November 2013 Accepted: 27 January 2014 Published: 07 Feb 2014}

\section{References}

1. Wiman, A: Uber die reellen losungen der linearen Differentialgleichungen zweieter ordnung. Ark. Mat. Astron. Fys. 12 14 (1917)

2. Bohner, M, Peterson, A: Dynamic Equations on Time Scales: An Introduction with Applications. Birkhäuser, Boston (2001)

3. Hilger, S: Analysis on measure chains - a unified approach to continuous and discrete calculus. Results Math. 18, 18-56 (1990)

4. Hartman, P, Wintner, A: The asymptotic arcus variation of solutions of real linear differential equations of second order. Am. J. Math. 70, 1-10 (1948)

5. Potter, RL: On self-adjoint differential equations of second order. Pac. J. Math. 3, 467-491 (1953)

6. Nehari, Z: Oscillation criteria for second order linear differential equations. Trans. Am. Math. Soc. 85, $428-445$ (1957)

7. Gingold, H: On the location of zeros of oscillatory solutions. Trans. Am. Math. Soc. 279, 471-496 (1983)

8. Swanson, CA: Comparison and Oscillation Theory of Linear Differential Equations. Academic Press, New York (1968)

9. Anderson, DR, Zafer, A: Nonlinear oscillation of second-order dynamic equations on time scales. Appl. Math. Lett. 22, 1591-1597 (2009)

10. Erbe, L, Peterson, A, Rehak, P: Comparison theorems for linear dynamic equations on time scales. J. Math. Anal. Appl. 275(1), 418-438 (2002)

11. Erbe, L, Peterson, A, Saker, SH: Oscillation criteria for second-order nonlinear dynamic equations on time scales. J. London Math. Soc. (2) 67(3), 701-714 (2003)

12. Leighton, W: On self-adjoint differential equations of second order. J. Lond. Math. Soc. 27, 37-47 (1952)

13. Kneser, A: Untersuchungen über die reellen Nullstellen der Integrale linearer Differentialgleichungen. Math. Ann. 42, 409-435 (1893)

14. Hovhannisyan, G: Asymptotic stability for dynamic equations on time scales. Adv. Differ. Equ. 2006, Article ID 18157 (2006)

\section{Submit your manuscript to a SpringerOpen ${ }^{\circ}$ journal and benefit from:}

- Convenient online submission

- Rigorous peer review

- Immediate publication on acceptance

- Open access: articles freely available online

- High visibility within the field

- Retaining the copyright to your article 